\title{
The Surface and Bulk Magnetic Properties of Fe-Al Alloys
}

\author{
A. HENDRYCH ${ }^{a, b, *}$, O. ŽIVOTSKÝ ${ }^{a, b}$, Y. JIRÁSKOVÁ ${ }^{c}$, I. MAŤKO ${ }^{d}$ \\ ${ }^{a}$ Department of Physics, ${ }^{b}$ Nanotechnology Centre, VŠB-Technical University of Ostrava, 17. listopadu 15/2172, \\ CZ-70833 Ostrava, Czech Republic \\ ${ }^{c}$ CEITEC IPM, Institute of Physics of Materials, AS CR, Žižkova 22, CZ-61662 Brno, Czech Republic \\ ${ }^{d}$ Institute of Physics, SAS, Dúbravská cesta 9, SK-84511 Bratislava, Slovak Republic
}

\begin{abstract}
The present paper is devoted to Fe-Al alloys with aluminium content of 28, 33 and 35 at.\%, prepared by standard technological procedure. The attention is focused on the comparison of the surface and bulk magnetic properties, which were obtained by magneto-optical Kerr effect (MOKE) and atomic and magnetic force microscopy (AFM/MFM) as the surface-sensitive methods combined with the vibration sample magnetometer (VSM) yielding the bulk magnetic properties. As was expected, the $\mathrm{Fe}_{72} \mathrm{Al}_{28}$ sample shows an overall ferromagnetic behavior at room temperature. Nevertheless, the bulk hysteresis loop shows single-phase behavior, while the surface MOKE hysteresis loop consists of two overlapped curves characterized by different coercivity values. The other two samples are paramagnetic from the viewpoint of the bulk, but reveal ferromagnetic behavior of their surfaces, represented by hysteresis loops, corresponding to rather harder magnetic phase. These results are well supported by MFM.
\end{abstract}

DOI: 10.12693/APhysPolA.126.58

PACS: 75.50.Bb, 75.70.Rf, 68.37.Rt, 78.20.Ls, 75.60-d

\section{Introduction}

The Fe-Al alloys attract ongoing interest of the wide scientific community [1]. The reason is their outstanding corrosion and oxidation resistance, good mechanical properties, low material costs and relatively large possibility of alloying with other elements or compounds as, e.g., with yttrium oxide in the known oxide dispersion strengthened alloys for high temperature applications $[2$, 3]. A fast advancing surface engineering predicates that the features and behavior of components are often influenced by their surface structure and physical properties. This stimulated the present investigations and concentrated the attention on the comparison of the surface and bulk magnetic properties. For this purpose three different compositions were chosen. The alloy with 28 at.\% $\mathrm{Al}$ belongs to the iron rich region, showing the ferromagnetic behavior at room temperature, while the alloys with 33 and 35 at.\% Al should be paramagnetic [4].

\section{Experimental}

All Fe-Al alloys were prepared from the pure $\mathrm{Fe}$ and Al $(99.99 \%)$ elements by induction melting. The ingots were cut into the round samples, approximately $10 \mathrm{~mm}$ in diameter and $500 \mu \mathrm{m}$ thick. Their surfaces were grinded and finely polished up to mirror finish. The surface and bulk hysteresis loop measurements were carried out at room temperature by the magneto-optical Kerr effect (MOKE) and vibrating sample magnetometer (VSM),

\footnotetext{
* corresponding author; e-mail ales.hendrych@seznam.cz
}

respectively. Details concerning the MOKE measurements can be found in Ref. 5. The bulk hysteresis loops were measured using VSM MicroSense in an external field of $\pm 1600 \mathrm{kA} / \mathrm{m}$. AFM $/ \mathrm{MFM}$ images were obtained by NTEGRA Prima platform operated in semi-contact mode. The tips used for measurements were coated with Co-Cr magnetic film.

\section{Results and discussion}

Figure 1a represents the surface MOKE hysteresis loops of the $\mathrm{Fe}-\mathrm{Al}$ alloys with aluminum content of 28 , 33 and 35 at.\%. It documents a ferromagnetic behavior of all samples. The $\mathrm{Fe}_{72} \mathrm{Al}_{28}$ sample surface embodies more complex structure with two magnetically different phases with different coercivity values, of approximately 5 and $17 \mathrm{kA} / \mathrm{m}$, respectively. Both values are substantially higher compared to the bulk value of $0.06 \mathrm{kA} / \mathrm{m}$. This is due to more inhomogeneous surface structure consisting of $\mathrm{Fe}-\mathrm{Al}$ phase and very probably oxide phase both significantly influenced by stresses and defects induced by the grinding and polishing. The induced stress relief causes also a ferromagnetic behavior of the next two alloys, $\mathrm{Fe}_{67} \mathrm{Al}_{33}$ and $\mathrm{Fe}_{65} \mathrm{Al}_{35}$ at their surfaces contrary to bulk paramagnetic behavior. As the hysteresis loops, measured by VSM, document in Fig. 1b, the magnetic moment induced by the applied field is nearly linear with the field strength and is rather weak. Nevertheless, the detailed view of this dependence around the zero applied fields in Fig. 1c, yields very small remnant magnetization and large coercivity. In contrast, the surface hysteresis loops of both samples are prominent. They mainly differ in the magnitude of the magneto-optical effect, that is at the $\mathrm{Fe}_{65} \mathrm{Al}_{35}$ sample surface approximately three times smaller. It is due to many factors influencing the surface properties, such as the element composition of the Fe-Al phase and its depth homogeneity, surface morphology, oxidation, defects, etc. That should be specified in 
the following investigations together with their individual contributions in more details. The magnetic behavior at both sample surfaces was confirmed by the MFM. The picture for the $\mathrm{Fe}_{65} \mathrm{Al}_{35}$ sample is seen in Fig. 2 (right) simultaneously with the surface topography reflected in the AFM result in Fig. 2 (left). Contrary to the surface of the $\mathrm{Fe}_{72} \mathrm{Al}_{28}$ sample where nearly invisible differences in color contrast were observed, the picture taken from the surface of the $\mathrm{Fe}_{65} \mathrm{Al}_{35}$ sample has yielded an interesting color relief. This is in agreement with the Mössbauer spectrometry using conversion electrons (not presented here), yielding higher fraction of paramagnetic phase in the $\mathrm{Fe}_{65} \mathrm{Al}_{35}$ sample surface.
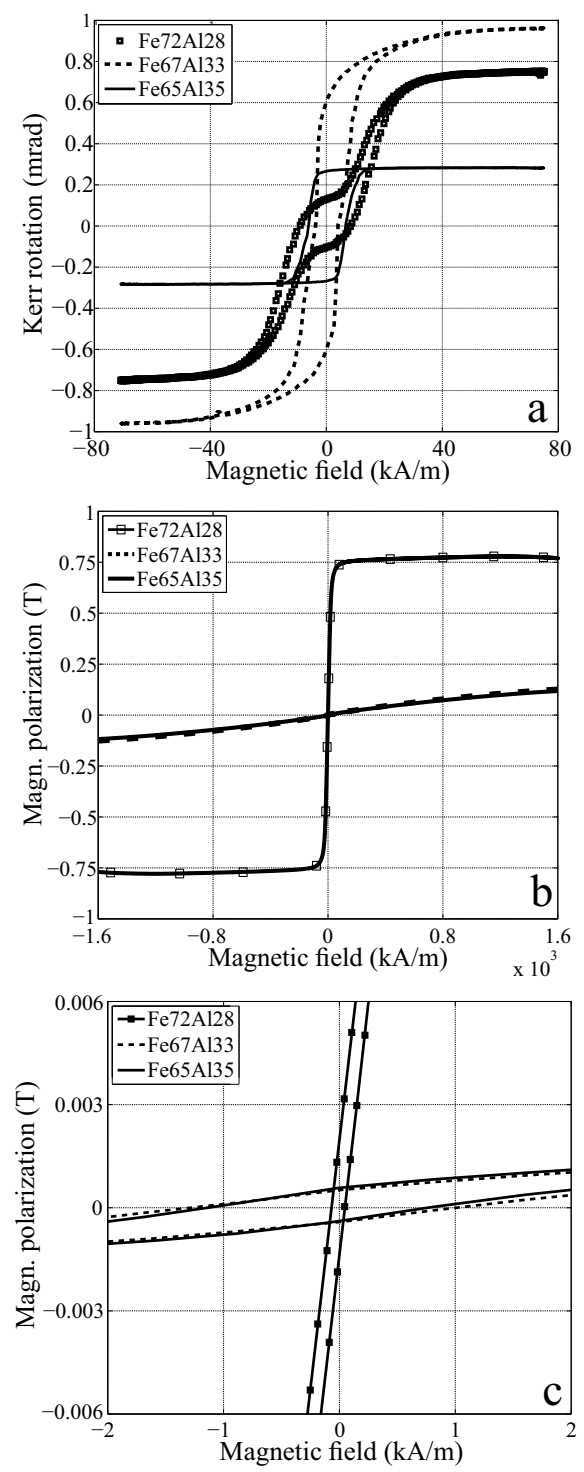

Fig. 1. MOKE hysteresis loops (a) and VSM hysteresis loops (b) with a detailed view around small applied fields (c) of the $\mathrm{Fe}-\mathrm{Al}$ samples with denoted $\mathrm{Al}$ compositions.
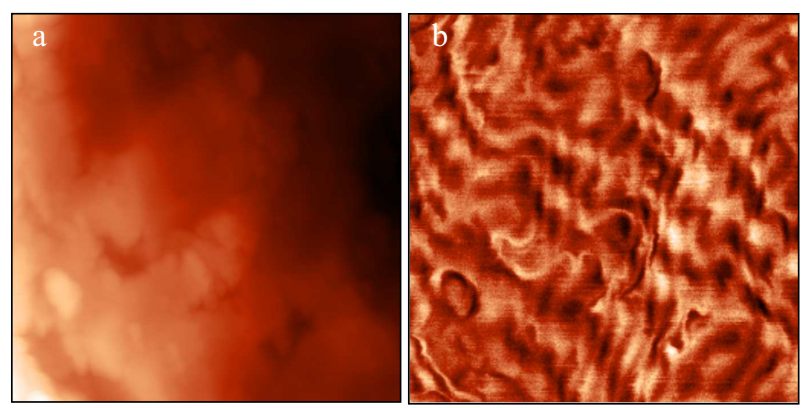

Fig. 2. $10 \times 10 \mu \mathrm{m}^{2}$ AFM (a) and MFM (b) images of $\mathrm{Fe}-\mathrm{Al}$ alloy with aluminium content of 35 at.\%.

\section{Conclusions}

The present investigations are devoted to the surface and bulk properties of the still important and frequently studied technical material of the Fe-Al composition. A difference in the bulk and surface magnetic behavior, caused by the several mechanical surface treatments such as grinding and polishing, is evidenced. Whereas the bulk magnetic properties of the as-prepared samples at room temperature are in agreement with phase diagram yielding ferromagnetic behavior for 28 at.\% Al and paramagnetic behavior for the samples with 33 and 35 at.\% $\mathrm{Al}$, the surface magnetic properties differ and show transformation into ferromagnetic state also in samples with higher $\mathrm{Al}$ content.

\section{Acknowledgments}

This work was supported by IT4 Innovations Centre of Excellence project, reg. no. CZ.1.05/1.1.00/02.0070, by the Grant Agency of the Czech Republic (P205/11/2137), by the grant SGS (SP2013/51), by the Ministry of Education, Youths and Sports of the Czech Republic (project 7AMB12SK009), and by Slovak Research and Development Agency (project APVV SK-CZ-0096-11).

[1] A. Hotař, M. Palm, Intermetallics 18, 1390 (2010).

[2] D.G. Morris, M.A. Muňoz-Morris, C. Baudin, Acta Mater. 52, 2827 (2004).

[3] M. Karlík, P. Haušild, V. Šíma, P. Málek, T. Vlasák, Int. J. Mater. Res. 100, 386 (2009).

[4] L.H. Bennett, T.B. Massalski, J.L. Murray, H. Baker, Binary Alloy Phase Diagrams, Ed. T.B. Massalski, American Society for Metals, Metals Park (OH) 1986.

[5] Y. Jirásková, A. Hendrych, O. Životský, J. Buršík, T. Žák, I. Procházka, D. Janičkovič, Appl. Surf. Sci. 276, 68 (2013). 